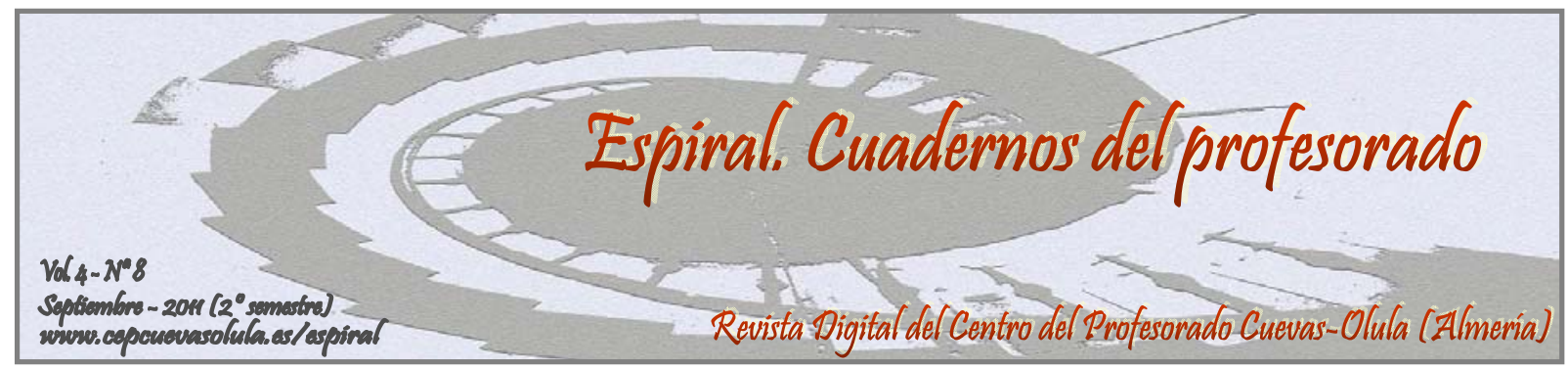

\title{
LAS TIC'S Y LAS VIÑETAS: UNA PROPUESTA DIDÁCTICA SOBRE LOS TOTALITARISMOS A TRAVÉS DEL CÓMIC MAUS
}

\section{ICT'S AND BULLETS: A PROPOSAL ON TEACHING THROUGH COMIC TOTALITARIANISM MAUS}

Ignacia Berenguel Aguilera

\section{Licenciada en Humanidades}

RESUMEN: En el presente trabajo planteamos una propuesta didáctica para la materia de Ciencias Sociales, Geografía e Historia dirigida al cuarto curso de Educación Secundaria Obligatoria basada en una metodología de interacción entre iguales, como eje principal del proceso de enseñanzaaprendizaje. En ella incluimos todos los elementos curriculares propios de una unidad didáctica, así como la propuesta de diferentes actividades para realizar en el aula-clase. Partimos de la lectura del cómic "Maus, retrato de un superviviente" de A. Spielgman, a partir de la cual, y haciendo uso de las Nuevas Tecnologías, el alumnado analizará, investigará y conocerá de un modo diferente, visual y complementario el horror del Holocausto y las calumnias de Auschwitz.

Palabras clave: cctividades cooperativas, Holocausto, cómic, unidad didáctica, ciencias sociales.

ABSTRACT: The present work develops a didactic proposal for the area of Social Sciences, Geography and History aimed to the fourth Course of Secondary Obligatory Education and based on an interactive methodology among peers, as the main point of the teaching-learning process. It is also included all the core curricular elements required in a Didactic Unit, as well as a wide range of activities to be developed in the classroom. Starting with the comic " Maus, portrait of a survivor" as the reading material; and making use of the New Technologies, students will be able to analyze, investigate and know the frightfulness about the Holocaust and the slanders of Auschwitz, in a different, visual and complementary way.

Key words: cooperative activities, Holocaust, comic, didactic unit, social sciences.

Berenguel Aguilera, I. (2011). Las Tic's y las viñetas: una propuesta didáctica sobre los totalitarismos a través del cómic Maus. Espiral. Cuadernos del Profesorado, 4(8), 42-57. Disponible en: http://www.cepcuevasolula.es/espiral.

Fecha de recepción: 27/05/2011

Fecha de aceptación: 15/08/2011
Enviar correspondencia a: ignaciaberenguel@orangemail.es 


\section{1.- INTRODUCCIÓN}

Una de las unidades didácticas que proponemos para desarrollar en cuarto curso de la Educación Secundaria Obligatoria es "El Holocausto judío". En el tema, trataremos contenidos de cierta complejidad para el alumnado de esta etapa, tales como limpieza étnica, socialdemocracia o "solución final”, por lo que nos hemos decantado por buscar metodologías dinámicas y por ende, a la utilización de medios y recursos didácticos susceptibles de despertar un elevado grado de atención entre el alumnado, consiguiendo así aprendizajes significativos, relevantes y motivadores, tal y como la Ley Orgánica 2/2006 de Educación (LOE) y la Ley 17/2007, de Educación de Andalucía (LEA) indica. Por lo tanto, creemos que nos encontramos ante el marco idóneo para llevar a la práctica actividades cooperativas, desde donde podemos beneficiarnos de numerosas ventajas, favoreciendo además uno de los principales retos de la educación: el desarrollo de actitudes básicas para una convivencia adecuada en sociedad.

Siguiendo a Martínez-Salanova (1998), una educación participativa que intenta aproximarse a la realidad del entorno social de los alumnos y alumnas no puede ignorar la importancia que supone el uso de los diferentes medios de comunicación y los valores didácticos que éstos aportan como forma de integración de la enseñanza en el contexto cotidiano. Por ello, nuestra propuesta se centra en el análisis del cómic Maus, relato de un superviviente, y en la realización de una webquest sobre dicho relato. Desarrollamos por lo tanto una estrategia de aprendizaje que potencia el desarrollo de las capacidades intelectuales del alumnado. Estas capacidades a su vez se encuentran estrechamente relacionadas con el área de Ciencias Sociales, donde utilizaremos Internet como fuente de información principal, integrando así, las Tecnologías de la Información y la Comunicación en el currículum de Secundaria.

\section{Mauss, retrato de un superviviente.}

Maus se presenta como una de las mayores obras en la historia del cómic. Publicada en dos tomos, y traducida a múltiples idiomas fue premio Pulitzer en 1992. Su autor, Art Spiegelman, nació en 1948 en Estocolmo donde se habían establecido sus padres, tras haber pasado por los campos de concentración nazis. Posteriormente, en 1951, se trasladan a Nueva York. En 1978, crearía junto a su esposa Francoise Mouly, la revista Raw, sinónimo desde entonces de vanguardia en el mundo del cómic. Será dentro de ella donde, tras trece años de reunir información, dibujar y preparar el guión, nacerá Mauss.

Spiegelman describe en Maus, la historia de su propio padre marcado por su terrible experiencia como superviviente del Holocausto. Se relata tal y como le fue contada al autor, quién la transcribió en imágenes trasladándola al mundo del cómic.

La obra comienza con la historia de amor entre Anja, hija de familia acomodada, y Vladek, joven empresario polaco (la madre y el padre del autor) en la época de dominio fascista y nacionalsocialista europeo de los años treinta. Al poco tiempo de casarse comenzará la persecución de los judíos por los nazis, iniciándose así, una serie de problemas que tendrán que superar con muchas dificultades. En primer lugar serán separados en guetos, a continuación tendrán que esconderse en distintos lugares, donde poco a poco van perdiendo a sus familiares (incluido a su pequeño hijo Richieu) y, por último, serán llevados al campo de concentración nazi de Auschwitz-Birkenau. Allí, tratados como animales y utilizados como esclavos, tendrán que usar todos los trucos posibles para evitar terminar en las cámaras de gas. Al final de la guerra Vladek y Anja se reencuentran. Sin embargo, lo vivido los marca para siempre, Anja se suicida en 1968 y a Vladek le queda un carácter huraño y difícil de tratar.

Maus representa un testimonio fiel del mayor genocidio que ha vivido nunca la historia humana, un hecho para el que no existen calificativos y que provoca en el lector rabia y tristeza.

El carácter innovador de Maus dentro del mundo del cómic se aprecia, en primer lugar, por la propia estructura de la historia, relatada combinando dos planos: el primero (presente), el hijo nos relata la compleja relación que mantiene con su padre, mientras que recoge notas para la realización de un cómic que contará las vivencias de aquel en la guerra; en el segundo (pasado-presente), asistimos a la historia en sí, la de un joven inmerso en el infierno nazi. Otra novedad a destacar se centra en la 
originalidad de la caracterización de los personajes, reflejados en papel como especies de animales diferentes en función de su nacionalidad y condición: los judíos son ratones, los alemanes son gatos y los polacos son cerdos.

\section{Maus y las competencias básicas}

La normativa actual tanto la LOE (2006) como la LEA (2007) contempla que el currículo de la Educación Secundaria Obligatoria se plasmará en aprendizajes significativos, relevantes y motivadores para el alumnado y se orientará a adquirir las competencias básicas, es decir, desarrollar destrezas, habilidades y actitudes que le resulten útiles para desenvolverse de forma autónoma en la vida diaria. Dado el carácter integrador del cómic Maus, retrato de un superviviente y la utilización de las Nuevas Tecnologías de la Información y la Comunicación, junto al desarrollo de las diferentes actividades, estrategias metodológicas, organización del aula y evaluación, nuestra propuesta contribuye a la adquisición de las siguientes competencias básicas:

a) Competencia en comunicación lingüística. En nuestra propuesta, esta competencia se adquiere a través de la propia lectura del cómic, permitiendo al alumnado conocer y usar nuevos términos, enriqueciendo así su vocabulario (búsqueda en el diccionario de términos y emplearlos de forma correcta, comprensión de las lecturas, etc.); y por el debate y exposiciones del trabajo final, contribuyendo a expresarse adecuadamente y respetar opiniones diferentes a la propia.

b) Conocimiento e interacción con el mundo físico. En el cómic aparecen distintos espacios geográficos como Polonia y Alemania, en los que se han desarrollado distintos episodios de la historia del siglo XX como: la Segunda Guerra Mundial, el Holocausto judío, creación de campos de concentración nazis, etc. Se pretende, pues, que el alumnado, a partir de la herramienta Google-maps utilice mapas, planos y localice en ellos los espacios que aparecen en el relato relacionándolos con los hechos históricos descritos, adquiriendo así, procedimientos de orientación, relación y localización.

c) Tratamiento de la información y competencia digital. A partir del desarrollo de la webquest, el alumnado tendrá que buscar, seleccionar y analizar información obtenida a través de internet, tanto para adquirir nuevos conocimientos como para ampliar los ya adquiridos. Con ello, el discente adquirirá destrezas y habilidades básicas digitales del trabajo tecnológico.

d) Competencia social y ciudadana. El clima cooperativo que pretendemos desarrollar en el aula será propicio para crear un ambiente de respeto y amistad, permitiendo así que el alumnado pueda expresar sus opiniones de forma asertiva, escuchando y comprendiendo distintos puntos de vista, así como la reflexión de forma crítica sobre las causas y consecuencias del Holocausto.

e) Competencia cultural y artística. A través de la lectura del cómic propuesto, de las lecturas complementarias y la visualización de fragmentos de películas, con sus debates, análisis y el trabajo de investigación, el alumnado podrá conocer fundamentalmente las manifestaciones artísticas y culturales de la comunidad judía, permitiéndole así, enriquecerse con las diferentes realidades y producciones y tomar conciencia de la necesidad de conservar el patrimonio cultural.

f) Competencia para aprender a aprender. La estructura de la propuesta que presentamos (actividades, organización de grupos, responsabilidades del alumnado, etc.) favorecerá al alumnado en la adquisición de hábitos de trabajo, la organización y distribución del tiempo, la realización de consultas, relacionar los nuevos contenidos con otros anteriores, etc. Con ello, estaremos favoreciendo el desarrollo del saber comunicar y expresar de forma efectiva los resultados del propio trabajo, la posibilidad de buscar explicaciones multicausales así como la toma de conciencia sobre lo que se ha aprendido y lo que el alumnado investigará por su cuenta. 
g) Autonomía e iniciativa personal. Nuestra propuesta permitirá al alumnado ser responsable de sus propias tareas y conocer el por qué y el para qué de los trabajos. Con ello se favorecerá el desarrollo de iniciativas de planificación y ejecución de estrategias, para comprender y resolver de la manera más adecuada las actividades planteadas, el saber argumentar de forma lógica y coherente los propios puntos de vista, así como la toma decisiones.

\section{Objetivos y contenidos de trabajo}

Partiendo del Real Decreto 1631/2006, de 29 de diciembre y en concordancia con los objetivos de área y de etapa que se establecen, Maus, ofrece numerosas posibilidades para alcanzarlos. Concretamos, pues, para esta propuesta didáctica los siguientes objetivos:

1. Conocer El Holocausto como un punto decisivo en la historia de la humanidad.

2. Localizar e identificar en un mapa los campos de concentración nazis más importantes de Europa, y estudiar su funcionamiento.

3. Emplear adecuadamente un vocabulario propio de la materia.

4. Reflexionar sobre la denominada "limpieza étnica” hasta nuestros días.

5. Explicar las consecuencias de la ideología extremista de los regímenes totalitarios.

6. Realizar una webquest mediante el trabajo en equipo construyendo nuevos aprendizajes a través de la búsqueda y selección de información.

7. Concienciar al alumnado sobre las consecuencias del uso y abuso del poder.

8. Contribuir al Plan de lectura del centro a través del trabajo con cómics.

9. Potenciar el desarrollo de habilidades sociales cooperativas, la creatividad y valores como el respeto, tolerancia, la igualdad y libertad.

Los medios por los cuales pretendemos alcanzar los objetivos propuestos, basándonos en lo exigido en el Real Decreto antes mencionado, los contenidos de Maus, relato de un superviviente, están íntimamente relacionados con el currículum de $4^{\circ}$ de E.S.O., aunque son susceptibles de extrapolarse a otros, como Historia del mundo Contemporáneo de $1^{\circ}$ de Bachillerato. podrían ser:

Así, desde esta perspectiva, los contenidos que podemos trabajar con nuestro alumnado,

1. El holocausto judío. Los campos de concentración nazis: Auschwitz Birkenau.

2. Valoración del cómic como reflejo de la situación política y social del momento.

3. Utilización de las tecnologías de la información y la comunicación (TIC's) de forma autónoma para localizar, seleccionar y organizar información.

4. Estímulo en el alumnado de hábitos de investigación, motivación y lectura.

5. Desarrollo de una actitud crítica respecto a cualquier forma de discriminación e intolerancia.

6. Análisis de las causas del Holocausto.

7. Lectura, interpretación, comentario y síntesis de diferentes textos relacionándolos con la actualidad.

8. Elaboración de un trabajo de investigación sobre la vida en un campo de concentración nazi.

En la presente propuesta didáctica planteamos como objetivo general que el alumnado consiga un conocimiento significativo y relevante de los totalitarismos y del Holocausto judío como un acontecimiento trascendente dentro del siglo XX, partiendo del análisis del cómic y trabajando de forma cooperativa con las TIC. 


\section{2.- METODOLOGÍA}

Entendemos por Metodología el conjunto de criterios, decisiones que organiza, de forma global, la acción didáctica en el aula, es decir, el papel que desempeña el alumnado y profesorado, utilización de medios, recursos, tipos de actividades, organización de los tiempos y espacios, agrupamientos, etc. La introducción de las competencias básicas en el currículo de la ESO en la LOE nos invita a cambiar y buscar nuevas metodologías.

En nuestra propuesta, siguiendo el Decreto 231/2007 de 31 de julio, en el que nos propone que la metodología de aula será fundamentalmente activa y participativa favoreciendo el trabajo individual y cooperativo del alumnado, y teniendo en cuenta que nuestras aulas presentan una gran diversidad de alumnado, nos basamos en el aprendizaje cooperativo. Para Johnson, Johnson y Holubec (1999), el aprendizaje cooperativo se basa en el empleo didáctico de grupos reducidos en los que las alumnas y los alumnos trabajan juntos para maximizar su propio aprendizaje y el de los otros. La cooperación consiste en trabajar juntos para alcanzar objetivos comunes.

Proponemos por tanto, trabajar con una estructura grupal heterogénea, formando grupos de cuatro o cinco alumnas y alumnos, repartidos en función de su nivel de competencia curricular, género, etnia y actitud, intentando que los equipos sean lo más similares posible. Con ello, y siguiendo a Pujolás (2009) contribuimos a potenciar las relaciones positivas entre iguales y promoveremos la participación activa del alumnado, ya que éste tendrá la oportunidad de convivir dentro de un grupo heterogéneo, trabajar junto a sus compañeros y compañeras de equipo, ayudándose y cooperando, conociéndose mejor y aceptándose y respetándose mutuamente. Además, el alumno o alumna, a través de esta forma organizativa, aprenderá cuestiones nuevas de sus compañeras y compañeros, rectificando, consolidando o reafirmando sus aprendizajes.

De este modo, emplearemos diversas técnicas metodológicas cooperativas, con el objeto de atender a los diferentes ritmos de aprendizaje del alumnado. Aplicaremos técnicas simples como "lápices al centro” o "lectura compartida”, y otras más complejas como "los grupos de investigación”.

\section{Recursos y materiales}

La selección de los materiales y recursos didácticos se presenta como una de las acciones que va a repercutir de forma más directa dentro de la relación del alumnado con los contenidos de enseñanzaaprendizaje y con las diversas formas de acceder a los mismos, de ahí que se contemple esta fase como un ejercicio de gran importancia y trascendencia.

Como hemos indicado anteriormente, pretendemos que el alumnado consolide lo aprendido desde un punto de vista dinámico, motivador, despertándole la capacidad de búsqueda y mejorando el proceso lecto-escritor. Por ello, el cómic y las Nuevas Tecnologías de la Información y la Comunicación (TIC's) se convierten en los ejes principales en nuestra propuesta, cuyas aplicaciones y ventajas didácticas son innumerables, como así ha quedado ampliamente demostrado por diversos autores como J.L. Rodríguez Diéguez (1988) “el tebeo es un modo de expresar acontecimientos, siendo algo más que un lenguaje, pues se trata de un medio de contar la Historio o de analizar tendencias culturales", o González et al. (2004) "Las nuevas aplicaciones tecnológicas constituyen un poderoso instrumento para estimular el aprendizaje e incrementar la calidad de la enseñanza”. A lo que le añadimos, además, la gran versatilidad didáctica para la educación de nuestros días en cuanto a la adquisición de las competencias básicas y los contenidos fijados en el currículum actual de la Enseñanza Secundaria Obligatoria para la clase de Ciencias Sociales, Geografía e Historia.

A partir de la lectura guiada, análisis del cómic y de la realización de la webquest, el alumnado conocerá, analizará e investigará, de un modo diferente, el Holocausto judío y cómo éste ha sido un punto decisivo en el siglo XX y en la historia de la humanidad, reflexionando a su vez, sobre derechos humanos, y conceptos tales como discriminación, prejuicios, racismo, tolerancia y genocidio.

Utilizaremos además, mapas, atlas, diccionarios de términos históricos y geográficos, enciclopedias, proyección de películas y lecturas complementarias (adscritas al Plan de Lectura del Departamento y del Centro). Todo ello se encontrará a disposición del alumnado en la biblioteca del 
centro, en el Departamento de Geografía, Historia e Historia del Arte y en el blog de clase de Ciencias Sociales.

\section{Educación en valores y contenido de tratamiento transversal}

Actualmente necesitamos una enseñanza integral, que incorpore contenidos de actualidad a las Ciencias Sociales. Para lograrlo, nos apoyaremos en una serie de contenidos de tratamiento transversal, cuya finalidad contribuirá a que nuestro alumnado consiga actitudes vitales que favorezcan una actitud integral de su personalidad, (Llopis 1996).

La LEA en su art. 39 y en el art. 5 del Decreto 231/2007, recoge y formula principios para "La educación en valores". Estos contenidos, que no son algo añadido, sino que suponen "poner la lupa" en determinados aspectos, variar el punto de mira e incorporar una nueva perspectiva, permiten al estudiante situarse en una sociedad caracterizada por la multiculturalidad. Siguiendo a Oscar Díez (1990) el cómic es un medio de persuasión, transmite valores éticos, ideológicos, es capaz de promover hacia una lectura crítica de la realidad. De ahí, el favorecer la reflexión y el análisis crítico por parte del alumnado, Maus, se convierte así en un material muy interesante para trabajar los valores y la interculturalidad.

El contenido del cómic seleccionado, nos permite desarrollar una transversalidad que abarca ámbitos como los que siguen:

- El fortalecimiento del respeto de los derechos humanos y de las libertades fundamentales y los valores que preparan al alumnado para asumir una vida responsable en una sociedad libre y democrática. Este aspecto, de vital importancia en nuestra propuesta, se desarrollará en las actividades de debate, análisis y reflexión acerca de las actitudes de las SS sobre la población judía principalmente, adoptando una actitud crítica ante los hechos que sucedieron. Se tratan, además, temas como la destrucción del hombre y el despojo de la identidad.

- Educación para la salud. En el cómic aparecen diversas viñetas en las que los personajes se encuentran en situaciones de salubridad lamentables, (hacinados en barracones, olores putrefactos, etc.) que provocan enfermedades como el tifus, lo cual permite la puesta en valor de la importancia de adquirir hábitos de vida saludable, y por otro, la capacidad de superación de los personajes ante las adversidades vividas.

- Aspectos de educación vial y salud laboral. Proponemos, en cuanto al primero, un estudio del viaje que realizan los prisioneros hasta la llegada al campo y de los trenes, como medio de transporte empleado. Y para el segundo aspecto, tratar el tema del trabajo esclavo (condiciones laborales, inclemencias meteorológicas, etc.).

- Coeducación. Nuestra propuesta es muy sugerente para trabajar las diferencias de trato que reciben en el campo los personajes según se trate de hombres o mujeres, la separación física en barracones o los roles establecidos. Éstos últimos los trabajamos a partir del personaje de Anja, dedicada a las labores del hogar y los demás a partir del propio análisis del cómic y en la realización del trabajo final de la webquest.

- Formación para la utilización de las nuevas tecnologías de la información y la comunicación. Su uso en nuestra propuesta es fundamental para la búsqueda y selección de información para la elaboración de la webquest que planteamos sobre la vida diaria en el campo de exterminio nazi.

Dado el carácter interdisciplinar que ofrece Maus, se trabajan contenidos de forma interrelacionada con distintas áreas como Lengua Castellana y Literatura, Lengua Inglesa, Educación Física, Educación Plástica y Visual, consiguiendo así un aprendizaje global e integrador.

- Educación Física. El alumnado, a partir de la visualización total o de fragmentos del documental Olimpia de Leni Riefenstah, podrá conocer Los Juegos Olímpicos de Berlín de 1936, e investigar sobre diversas cuestiones como por ejemplo: sobre el atleta Jesse Owens, o cómo se referían, despectivamente, a los atletas negros en el entorno de Hitler. 
- Música. Proponemos trabajar la obra "Un superviviente en Varsovia" de A. Schonberg, sobre la que el alumnado podrá realizar una audición acompañada de la letra y analizar la obra desde un punto de vista musical e histórico.

- Educación Plástica y Visual. El alumnado podrá conocer las características principales de un cómic a partir del análisis formal de las viñetas: composición, planos, tratamiento de la luz, etc., además de realizar comics sencillos resaltando algunos de los valores trabajados.

- Lengua extranjera inglesa. En la siguiente página web: www.ushmm.org/wlc/es/media_nm.php?MediaId=6366, podemos encontrar un vídeo muy interesante en inglés, a partir del cuál y guiados por el profesorado, el alumnado podrá trabajar imagen, texto y sus traducciones correspondientes.

- Lengua Castellana y Literatura. De forma coordinada con el profesorado de este departamento, realizaremos una tertulia dialógica literaria sobre el cómic, técnica que desarrollamos en el siguiente apartado.

\section{3.- DESARROLLO DE LA PROPUESTA}

\section{Actividades}

Las actividades constituyen un medio muy importante para el aprendizaje y configuran el contexto inclusivo idóneo para el desarrollo de las competencias básicas. Por ello, proponemos actividades motivadoras y que estimulen la atención del alumnado.

Para iniciar la sesión, hemos diseñado una Terturlia dialógica sobre el cómic "Maus, retrato de un superviviente". El objetivo es que, mediante el diálogo igualitario y las reflexiones individuales y grupales, el alumnado comprenda la importancia de lo que supuso el Holocausto y a la vez, que vayan desarrollando habilidades de comunicación y desarrollo con una actitud crítica respecto a cualquier forma de discriminación e intolerancia. El alumnado además, será capaz de interpretar la lectura, comentarla, sintetizarla y de relacionarla con valores tales como: tolerancia, responsabilidad, solidaridad, engaño, terror, sufrimiento.

Se le pedirá al alumnado, al menos con un mes de antelación a la fecha prevista del desarrollo de la unidad, que realice la lectura del cómic "Maus, retrato de un superviviente" indicándole a su vez que deberán de rellenar la siguiente ficha de trabajo (tabla 1):

Tabla 1.- "Maus, retrato de un superviviente"

Nombre y Apellidos:

Curso:

Fecha: Valoración:

Primera parte:

1. Tema principal:

2. Espacios en los que se desarrolla la historia:

3. Palabras clave:

4. Características y rasgos físicos de los protagonistas: Art, Vladek, Anja, Richieu, Mala y Françoise

Segunda parte:

1. Señala los párrafos o viñetas que te llamen la atención indicando el porqué:

Se les aconsejará que si lo desean podrán hacerlo en grupo, puesto que la actividad puede ser más enriquecedora y podrán surgir más preguntas para el trabajo y debate que realizaremos en clase posteriormente. 
En clase, en gran grupo se le dará respuesta a las preguntas de la primera parte y a continuación, cada discente explicará a sus compañeros y compañeras los párrafos o viñetas que ha señalado y los motivos de su elección. El profesor o profesora actuará como moderador estableciendo turnos de intervención, dejando que todos y todas aporten sus argumentos para que puedan reflexionar y discutir, dando prioridad a las personas que menos participen en la tertulia y reconduciendo el tema de discusión si se aleja demasiado de lo leído. Sería adecuado además, que propusiese vías de reflexión si se dejan sin tocar puntos relevantes, como por ejemplo:

- ¿Cómo es la bandera nazi? (Parte I, página 34)

- ¿Qué quiere decir “Ciudad libre de judíos”? (Parte I, página 35)

- ¿ ¿Qué significa la estrella de David? (Parte I, página 82)

- Reflexiona sobre el anuncio: "Recompensa, por cada judío no identificado que encuentre: 1 Kilo de azúcar” (Parte I, página 84).

- ¿Qué impacto debía de suponer para los prisioneros las alambradas, torretas de vigilancia, uniformes o barracones?

- ¿ ¿Por qué creéis que "En todo el lugar había un olor terrible... dulzón... como a goma quemada, o grasa”? (Parte II, página 87).

- ¿ ¿Por qué se les tatuaba a los presos el antebrazo? (Parte II, página 186)

- ¿ ¿Qué comían los presos en Auschwitz? (Parte II, página 209)

- ¿ ¿Qué os provoca “Te daban media ración de pan por una cuchara”? (Parte II, página 189)

- ¿ ¿Para qué las SS “pasaban revista” todos los días? (Parte I, página II, página 210)

- ¿¿Se beneficiaban los alemanes de las expropiaciones?

El grupo-clase, desde el inicio de la unidad y a lo largo de su desarrollo, dispondrá de un "saco de dudas". Se trata que cada discente o el portavoz del grupo escriba en un tercio de folio, indicando su nombre o el nombre del equipo, las dudas que se le planteen a lo largo del estudio del tema y la introduzca en una caja o urna. Al día siguiente el profesor o profesora saca una duda del "saco de dudas" y pide si alguien u otro equipo sabe resolverla, si no hay nadie que lo sepa, resuelve la duda el profesor o la profesora (Pujolás, 2009).

Como actividades de desarrollo y con la intención de que el alumnado aplique y consolide los contenidos trabajados, en pequeños grupos deberán de realizar las siguientes. Para ello podrán consultar el libro de texto, distintos diccionarios y la página Web de la Enciclopedia del Holocausto www.ushmm.org

1. Haz un comentario sobre el contexto histórico-geográfico en el que se desarrolla la historia de Maus.

2. ¿Qué es el Holocausto? Y ¿¿cuál es el origen de su “Negación”?

3. Busca en el diccionario o en la Web, el significado de las siguientes palabras: fascismo, nacionalsocialismo, totalitarismo, "solución final”, antisemitismo y gueto.

4. Explica la diferencia entre un campo de concentración y un campo de exterminio.

5. En los primeros años del régimen nazi, el gobierno nacionalsocialista estableció campos de concentración ¿Con qué intención?

6. ¿Qué eran "las marchas de la muerte”?

7. ¿Qué consecuencias tuvieron los crímenes cometidos durante el Holocausto para la comunidad judía?

Además, llevaremos a cabo una webquest sobre el campo de concentración Auschwitz Birckenau y el Holocausto judío, sobre el que versa la lectura analizada. A través de esta estrategia de investigación orientada, el alumnado a partir de una serie de recursos Web, aprenderá a trabajar en equipo, desarrollará habilidades de búsqueda y selección de información, analizará y reflexionará de 
forma crítica las causas y consecuencias del Holocausto judío, y utilizará las nuevas tecnologías de la información y la comunicación de una manera amena y motivadora.

\section{Desarrollo de la webquest “Auschwitz, el campo de la vergüenza” (figura 1)}

\section{Introducción}

A partir de la lectura que hemos realizado del cómic "Maus, historia de un superviviente", os propongo un viaje virtual al campo de concentración de Auschwitz-Birkenau, para conocer los lugares en los que estuvo la familia Spielgman.

Para ello te vamos a ayudar con esta investigación guiada a través de los apartados que tienes a continuación. En todas las páginas encontrarás, un menú secuencial que te irá llevando a cada uno de los apartados de esta actividad. Debes seguirlos en el mismo orden en el que aparecen, aunque en cualquier momento puedes ir a otros apartados de la WebQuest.

\section{Tareas}

Deberéis de analizar e investigar las preguntas que se os plantean y hacer una reflexión sobre el sentido que tiene recordar lo que fue el Holocausto, los peligros de los regímenes totalitarios y quiénes son hoy las víctimas. Todo ello quedará recogido en un trabajo final que

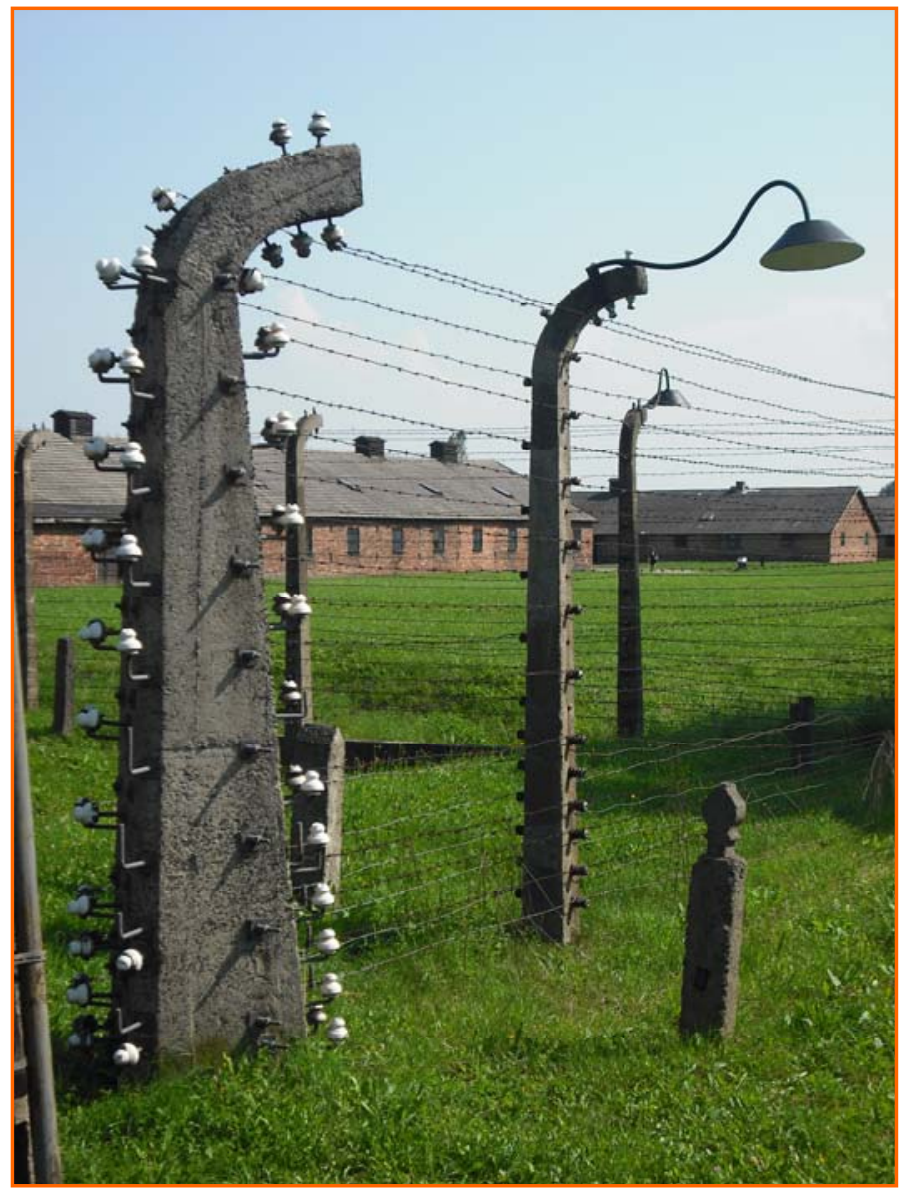

Figura 1.- Auschwitz, el campo de la vergüenza. Archivo personal deberéis de explicar al resto de la clase.

a. Miles de judíos (hombres, mujeres y niños) fueron asesinados en el campo de concentración de Auschwitz, pero ¿Quién más?

b. En la puerta de la entrada al campo de Auschwitz hay una inscripción “Arbeit macht frei”, ¿qué significa?

c. En las barracas solían apiñarse centenares de presos ¿qué consecuencias tenía?

d. Fíjate en los planos de Auschwitz (Plano I y II) ¿Qué diferencia hay?

e. ¿Cuánto tiempo estuvieron activos los crematorios y las cámaras de gas de Auschwitz?

f. ¿Dónde y quién procedía a la selección de los judíos cuando llegaban a Birkenau? ¿Cómo se efectuaba el acto de selección entre los judíos?

g. ¿Cuántos cadáveres se podían quemar diariamente en los hornos de los crematorios de Auschwitz?

h. En 1943 en la horca común que se encontraba en la Plaza de las revistas, las SS ahorcaron a doce presos políticos polacos ¿por qué?

i. ¿ ¿Por qué las SS en 1944 demolieron los hornos y el edificio del crematorio?

j. ¿Qué se utilizaba de forma rutinaria como medio para el exterminio en masa? 


\section{El proceso}

Para realizar el trabajo debes seguir los siguientes pasos:

a. Formarás un grupo de cuatro o cinco compañeras y compañeros con ayuda de la profesora.

b. Os debéis de organizar y repartir las tareas a realizar.

c. Cada grupo buscará, recopilará y analizará de forma ordenada la información necesaria para realizar el trabajo de investigación. Para ello, puedes utilizar tu ordenador con conexión a Internet, el libro de texto, atlas, la filmografía y lecturas recomendadas que encontrarás en el "Rincón del Holocausto" y toda la información que te ofrece la biblioteca del centro. Si lo consideráis oportuno fuera de horario escolar podéis buscar información en la biblioteca municipal. La información irá siendo recogida de forma ordenada para su posterior elaboración pero en todo momento, tienes que poner en común la información conseguida, los avances realizados y tendrás que hablar de temas controvertidos y llegar a un acuerdo sobre cómo y cuándo continuar el trabajo. No olvides que formas parte de un grupo de trabajo. Confía en tus compañeras y compañeros y permitid que ellas y ellos puedan confiar y apoyarse en ti.

d. Puesta en común de los resultados de la búsqueda. Todos los miembros del grupo expondrán ante los demás los resultados de su búsqueda particular, decidiendo de forma conjunta qué información se utilizará para confeccionar el documento final y cuál no.

e. Entre todos los miembros del grupo se llevará a cabo la elaboración definitiva de vuestro “documento" que se entregará a la profesora para que lo revise antes de la presentación.

\section{Recursos}

Para realizar el trabajo os propongo dos tipos de recursos:

a. Recursos en línea:

- El Campo de Concentración de Auschwitz 1940-1944:

- www.corazones.org/lugares/polonia/auschwitz/auschwitz_concentracion.htm

- www.nizkor.org/faqs/auschwitz/index-sp.html

- www1.yadvashem.org/es/multimedia/auschwitz_album.asp

- El Holocausto judío y los campos de concentración nazis:

o http://holocaustoyeducacion.es/index.html

o www.ushmm.org/wlc/es/

o www.segunda-guerra-mundial.com/

- Víctimas no judías del exterminio nazi:

o http://clio.rediris.es/fichas/Holocausto/exterminio.htm

- Andaluces exterminados en los campos de concentración nazi:

o www.foroporlamemoria.info/documentos/2005/mauthausen_maig2005.pdf

- Ley para la protección de la sangre y el honor alemanes: www.campodemarte.com

- Definición de términos: www.rae.es

b. Recursos fuera de línea:

- Spielgman, A. (2007). Maus, retrato de un superviviente. Barcelona: Reservoir Books.

- Levi, P. (1998): Si esto es un hombre. Barcelona: Muchnick.

- Acude a la biblioteca del centro y a la del Departamento social y de ciudadanía y echa un vistazo a lo que allí tienen que pueda ser útil para contestar a las preguntas, tales como: diccionario histórico-geográfico, artículos de revistas, Cd-Roms, etc. 


\section{Evaluación}

La evaluación será del grupo en cuanto a la forma en que fue producido el trabajo, pero también individual de acuerdo con el papel que cada uno desempeñó. Todo será evaluado, incluso como fue hecha la búsqueda y como el grupo logró alcanzar los objetivos.

\section{Créditos}

Esta actividad fue diseñada para el alumnado de $4^{\circ}$ de ESO, para la materia de Ciencias Sociales, Geografía e Historia.

Las actividades de cierre serán de síntesis y repaso de lo aprendido. En primer lugar, cada grupo presentará al resto de la clase su trabajo final de la Webquest, explicando sus reflexiones y argumentos. Y a continuación, para aclarar dudas, comprobar los contenidos aprendidos y hacer una síntesis de la unidad, se resolverán las dudas que queden en el "saco de dudas", repasando de forma general todas las que en él se depositaron.

Dada la diversidad natural de nuestro alumnado, y contando con diferentes ritmos de aprendizaje, a las tareas anteriores, añadimos actividades de refuerzo, diseñadas para el alumnado con dificultades en el proceso de enseñanza-aprendizaje; y de ampliación, enfocadas para aquel alumnado que avance más rápidamente en el desarrollo normal del currículo.

En cuánto a las primeras proponemos las siguientes:

1. Busca en la red o en el diccionario los siguiente términos, copia la definición en tu cuaderno de clase: Holocausto; Fascismo; Socialnacionalismo; Guetto; Antisemitismo; Gestapo; Kapo; "Solución final”

2. ¿Cuándo es el Día del Recuerdo del Holocausto?

3. Explica la diferencia entre un campo de concentración y un campo de exterminio.

4. Dibuja un mapa de Europa y señala lo siguiente:

- Los campos de concentración: Auschchwitz-Birkenau; Sachsenhausen y Mauthausen

- Ciudades y países: Alemania, Berlín, Cracovia, Polonia, Varsovia, Francia, Bélgica, Ucrania, Bielorrusia, y Lituania.

Y con respecto a las actividades de ampliación, las propuestas son:

1. Entre 1933 y 1939 se crearon por todo el territorio alemán campos de concentración bajo la jurisdicción exclusiva de las SS. A medida que Alemania iba avanzando en la conquista de Europa entre los años 1939 y 1941, las SS crearon varios campos de concentración nuevos en los que encerraba al creciente número de prisioneros políticos, grupos de resistencia y grupos considerados como razas inferiores, como los judíos y los romaníes (gitanos). Elige uno de estos campos e investígalo. Orientaciones para la investigación:

o En qué país se encuentra (sitúalo en un mapa).

o En qué año se construyó y cuánto tiempo estuvo funcionando como campo de concentración.

o Características del campo: superficie, modos de vida, instalaciones, etc.

2. Buscad información sobre los experimentos médicos, que el doctor Mengele llevó a cabo en Auschwitz, y haced un informe.

3. Lee con atención los siguientes artículos de la "Ley para la protección de la sangre y el honor alemanes" y contestad:

a. ¿Cuál es el enemigo nato de Alemania, en el pensamiento de los juristas y escritores nazis?

b. Comentar la concepción de la familia que aquí se proyecta y la ruptura de la intimidad del individuo, desde el momento en que pueden declarar nulo su matrimonio. 
c. Haced una reflexión sobre la tragedia que significó el Holocausto.

d. ¿ ¿iene sentido recordar, investigar lo que fue el Holocausto? ¿Quiénes son hoy las víctimas?

Tabla 2.- "Ley para la protección de la sangre y el honor alemanes” (extraído de www.campodemarte.com)

\section{"Ley para la protección de la sangre y el honor alemanes"}

Profundamente convencido de que la preservación de la sangre alemana es la condición primera de la supervivencia del pueblo alemán y animado de la irreductible voluntad de asegurar definitivamente el porvenir de la nación alemana, el Reichstag ha adoptado por unanimidad y promulga la siguiente ley:

\section{Articulo 1:}

Quedan prohibidos los matrimonios entre judíos y súbditos de sangre alemana o asimilada. Los matrimonios concertados a pesar de esta prohibición son nulos de todo derecho, incluso si, para burlar la ley, hubiesen sido contraídos en el extranjero.

Sólo el Procurador puede invocar una causa de nulidad

\section{Artículo 2:}

Quedan asimismo prohibidas las relaciones extraconyugales entre judíos y súbditos de sangre alemana o asimilada.

\section{Artículo 3:}

Queda prohibido que los judíos contraten como empleadas de hogar a mujeres de sangre alemana o asimilada de menos de 45 años.

\section{Artículo 4:}

Queda prohibido que los judíos enarbolen o engalanen con los colores nacionales.

En cambio, pueden engalanar con los colores judíos. El ejercicio de este derecho queda garantizado por el Estado.

\section{Artículo 5:}

Quien contravenga las disposiciones del artículo 1 puede ser sometido a reclusión.

El hombre que contravenga la disposición del artículo 2, puede ser sometido a reclusión.

Quien contravenga las disposiciones de los artículos 3 y 4, puede ser sometido a multa o a una pena de prisión de hasta un año.

\section{Artículo 6:}

El Ministerio del Interior del Reich, de acuerdo con el Representante del Führer, dictará las prescripciones legales y administrativas necesarias para la aplicación y publicación de la presente ley.

\section{Artículo 7:}

La presente ley entrará en vigor el día siguiente de su promulgación, excepto el artículo 3, cuya vigencia será a partir del $1^{\circ}$ de enero de 1936.

Nuremberg, 15 de septiembre de 1935.

El Führer y Canciller del Reich.

Para el desarrollo de la unidad, proponemos que el aula se organice de forma cooperativa. Para la terturlia dialógica, el alumnado se sentará en círculo y para el resto de actividades, las mesas y las sillas se dispondrán en forma de rectángulo, formando subgrupos, de forma que el alumnado se encuentre sentado perpendicularmente a la pizarra, (Pujolás, 2009).

Con la propuesta que aquí presentamos se contribuye con las orientaciones que establece el currículum de la ESO y el ROC en relación al Plan Lector, pero además, proponemos habilitar un 
"Rincón del Holocausto", para guardar todos los documentos tanto escritos (Auschwitz, El niño del pijama de rayas, Diario de Ana Frank, etc.), como audiovisuales (La vida es bella, La lista de Schindler, La Pasajera, El pianista, El gran dictador, etc.) que se utilicen en clase durante la realización del trabajo, no sólo los aportados por el profesorado sino también los creados por el alumnado o los que de forma voluntaria se aporte. Con ello se facilita el intercambio de obras e incluso la posibilidad de retomar alguna en un momento dado. La organización de este espacio, siguiendo a Romera y Martínez (2009), permite entre otros, que el alumnado se sienta más partícipe en el proceso de enseñanza-aprendizaje, valore sus progresos e implícitamente favorezcamos actividades de lectura y escritura.

\section{Actividades}

El proceso evaluador que llevaremos a cabo en esta propuesta, se realizará siguiendo las pautas que nos indica la Orden de 10 de agosto de 2007, y dirigido a todo el proceso de enseñanza-aprendizaje, lo que nos permitirá, según Zabala (2008), analizar y comprender todo lo que sucede en la acción formativa.

Tabla 3.- Valoración de la Webquest.

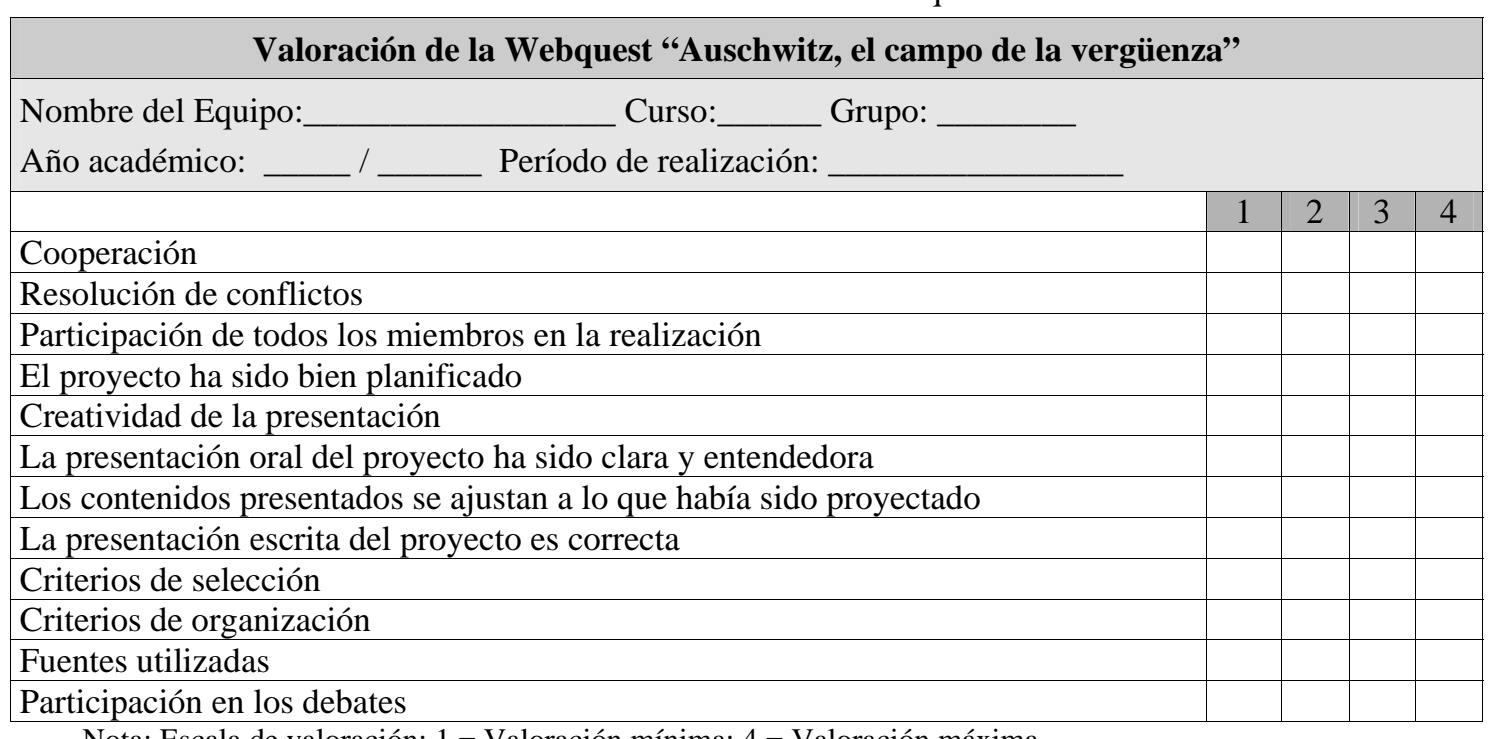

Nota: Escala de valoración: 1 = Valoración mínima; 4 = Valoración máxima

En estrecha relación con los Objetivos que nos hemos planteado para esta propuesta didáctica, proponemos sus Criterios de Evaluación, que constituyen el referente fundamental para valorar la consecución de los objetivos y el grado de adquisición de las competencias básicas.

- Explicar las causas y consecuencias del Holocausto judío y entender que constituye un punto decisivo en el siglo XX y en la historia de la humanidad.

- Definir fascismo, nacionalsocialismo, totalitarismo, "solución final”, antisemitismo y gueto.

- Saber las consecuencias del uso y abuso del poder y de la ideología extremista de los regímenes totalitarios.

- Conocer cómo viven los presos en los campos de concentración y de exterminio nazi y localizar en un mapa los más importantes.

- Adquirir y tomar conciencia de valores como el respeto, tolerancia, la igualdad y libertad.

La evaluación al ser un proceso continuo, integrador y flexible nos permite por un lado, plasmarla tanto de forma individual como grupal, y utilizar diversas técnicas e instrumentos de evaluación. 
Para la evaluación individual, utilizaremos por un lado, un registro anecdótico en el que se irá anotando, de forma continua, hechos o acontecimientos significativos de cada alumno o alumna (reflexiones, interés, expresión de ideas, etc.); por otro, la observación directa y sistemática, que nos permitirá valorar en el alumnado las intervenciones en clase, la participación en la tertulia, su implicación, el respeto de opiniones y de turnos de palabra; y el cuaderno de clase ya que nos arroja datos más específicos y detallados del aprendizaje de la unidad (anotaciones realizadas antes y durante la tertulia, realización y corrección de actividades, comprensión, ortografía, vocabulario utilizado, limpieza y organización del cuaderno).

Tabla 4.- Evaluación de las competencias básicas.

\begin{tabular}{|c|c|c|c|c|c|}
\hline Competencia & Indicadores & \multicolumn{4}{|c|}{$\begin{array}{c}\text { Grado de } \\
\text { adquisición }\end{array}$} \\
\hline \multirow{3}{*}{$\begin{array}{l}\text { Competencia en } \\
\text { Comunicación } \\
\text { lingüística }\end{array}$} & \multirow{3}{*}{$\begin{array}{l}\text { - Consigue una expresión oral y escrita adecuada } \\
\text {-Maneja vocabulario rico y creativo } \\
\text {-Comprende las lecturas realizadas }\end{array}$} & 1 & 2 & 3 & 4 \\
\hline & & 1 & 2 & 3 & 4 \\
\hline & & 1 & 2 & 3 & 4 \\
\hline \multirow{2}{*}{$\begin{array}{l}\text { Competencia en el } \\
\text { conocimiento y la } \\
\text { interacción con el } \\
\text { medio }\end{array}$} & \multirow{2}{*}{$\begin{array}{l}\text {-Comprende y explica el medio } \\
\text { •Utiliza mapas para orientarse }\end{array}$} & 1 & 2 & 3 & 4 \\
\hline & & 1 & 2 & 3 & 4 \\
\hline \multirow{2}{*}{$\begin{array}{l}\text { Competencia digital y } \\
\text { tratamiento de la } \\
\text { información }\end{array}$} & \multirow{2}{*}{$\begin{array}{l}\text {-Utiliza las fuentes de información correctamente } \\
\text { •Utiliza estrategias y habilidades básicas digitales para el } \\
\text { aprendizaje }\end{array}$} & 1 & 2 & 3 & 4 \\
\hline & & 1 & 2 & 3 & 4 \\
\hline \multirow{3}{*}{$\begin{array}{l}\text { Competencia social } \\
\text { y ciudadana }\end{array}$} & \multirow{3}{*}{$\begin{array}{l}\text {-Utiliza un lenguaje coeducativo, y no violento } \\
\text { - Trabaja en equipo, escuchando y respetando } \\
\text {-Participa de forma activa, responsable y solidaria en las } \\
\text { actividades de clase. }\end{array}$} & 1 & 2 & 3 & 4 \\
\hline & & 1 & 2 & 3 & 4 \\
\hline & & 1 & 2 & 3 & 4 \\
\hline \multirow{2}{*}{$\begin{array}{c}\text { Competencia artística y } \\
\text { cultural }\end{array}$} & \multirow{2}{*}{$\begin{array}{l}\text {-Dispone de habilidades de cooperación para contribuir a la } \\
\text { consecución de un resultado final } \\
\text { - Reelabora ideas propias y ajenas }\end{array}$} & 1 & 2 & 3 & 4 \\
\hline & & 1 & 2 & 3 & \\
\hline \multirow{3}{*}{$\begin{array}{l}\text { Competencia para el } \\
\text { desarrollo de la } \\
\text { autonomía personal }\end{array}$} & \multirow{3}{*}{$\begin{array}{l}\text { - Conoce el porqué y el para qué de los trabajos } \\
\text {-Es responsable de las tareas y producciones } \\
\text {-Planifica y organiza el trabajo en equipo }\end{array}$} & 1 & 2 & 3 & \\
\hline & & 1 & 2 & 3 & \\
\hline & & 1 & 2 & 3 & 4 \\
\hline \multirow{3}{*}{$\begin{array}{l}\text { Competencia para } \\
\text { aprender a aprender }\end{array}$} & \multirow{3}{*}{$\begin{array}{l}\text { - Relaciona los nuevos conceptos con los anteriores } \\
\text { - Aplica los conocimientos adquiridos en otras actividades. } \\
\text { - Adquiere hábitos de trabajo y estrategias de } \\
\text { aprendizaje. }\end{array}$} & 1 & 2 & 3 & 4 \\
\hline & & 1 & 2 & 3 & 4 \\
\hline & & 1 & 2 & 3 & \\
\hline
\end{tabular}

Nota: Poco: 1; Regular: 2; Adecuado: 3; Bueno: 4

Teniendo en cuenta la importancia que desde nuestra propuesta se le ha dado al trabajo cooperativo, la evaluación grupal tendrá una gran importancia realizándose con diversas estrategias metodológicas. Para evaluar las actividades de desarrollo se empleará la estrategia "Una para todos", es decir, la profesora recoge, al azar, el cuaderno de ejercicios de un miembro del equipo, lo corrige, y la calificación obtenida es la misma para todos los miembros de dicho equipo, fijada según el contenido de las respuestas a las actividades. Y para la valoración del trabajo final de investigación, la webquest, utilizaremos la estrategia "cabezas numeradas" (tabla 3). A todos los componentes del grupo se le asigna un número y a la hora de exponer el trabajo, la profesora nombrará un número al azar realizándolo el miembro del grupo que tenga dicho número. La nota de dicha exposición será global a todo el grupo. El hecho de desconocer qué alumno o alumna va a exponer el trabajo hace que cada uno tenga que comprender el trabajo realizado por todos y todas, (Pujolás, 2009). Para su correcta recogida de información hemos diseñado una tabla en la que se especifican los ítems a valorar.

En cuanto a la evaluación de las competencias básicas, proponemos una serie de indicadores de logro de las habilidades que el alumnado habrá adquirido al finalizar la unidad (tabla 4). 
Con los datos obtenidos podemos calificar al alumnado, por lo que proponemos los siguientes porcentajes evaluadores de carácter totalmente flexible e indicativo:

- Actividades y trabajo en grupo: $70 \%$

- Tertulia dialógica: $20 \%$

- Reflexiones sobre el Holocausto: $10 \%$

\section{4.- CONCLUSIONES}

El trabajo del cómic en el aula puede resultar un recurso muy valioso para el trabajo de educación en valores con el alumnado, siempre y cuando seleccionemos adecuadamente la obra y programemos las situaciones didácticas adecuadas.

El área de Geografía e Historia no puede quedar ajena a las Nuevas Tecnologías ya que se conforman como un recurso emergente muy valioso en el aula.

El uso de la webquest motiva al alumnado y lo convierte en protagonista del proceso de aprendizaje en la medida en que se le presentan propuestas de trabajo susceptibles de resolver por medio de la investigación.

Las técnicas de trabajo cooperativo potencian el desarrollo de las capacidades intelectuales. Por medio de la ayuda entre iguales representan una inestimable ayuda para el alumnado independientemente de su nivel de desarrollo curricular.

Las tertulias literarias se muestran como una magnífica estrategia para que el alumnado debata, muestre sus propias ideas y extraiga sus conclusiones en público. Por lo tanto, la programación de tertulias de forma regular ayuda a los jóvenes a comprender mejor la lectura debido en gran parte a la funcionalidad del método.

Una vez más, los contenidos del área de Geografía e Historia representan una oportunidad inestimable para educar en valores aprovechando acontecimientos significativos de la historia mundial.

\section{5.- REFERENCIAS BIBLIOGRÁFICAS}

Aguaded J. I. y Martínez-Salanova, E. (1998). Medios, recursos y tecnología didáctica para la Formación Profesional Ocupacional. Huelva: Facep.

Álvarez Méndez, J.M. (2001). Evaluar para conocer, examinar para excluir. Madrid: Morata.

Aparicio, R. (1992). El cómic y la fotonovela en el aula. Madrid: Ediciones La Torre.

Ballester, L. (2003). La Guía didáctica MAUS. Baleares: Inrevés.

Fernández, M. y Díaz O. (1990). El cómic en el aula. Madrid: Alhambra.

Gaumer, P. y Moliterni, C. (1994). Diccionario del cómic. Barcelona: Larousse Planeta.

González, R., Zabala, C., Fernández, O. y Wiliams, R. (2004). Aula modelo en el contexto de las Nuevas Tecnologías y Prácticas Educacionales (AMTEPE). Encuentro Educacional [en línea], 11 (2). Disponible en: http://www.revistas.luz.edu.ve/index.php/ed/article/view/322. Consulta [2010, 20 de noviembre].

Johnson D.W., Johnson R.T. y Holubec, E.J. (1999). El aprendizaje cooperativo en el aula. Buenos Aires: Paidós.

Ley Orgánica de Educación del 2/2006 de 3 de mayo. En Boletín Oficial del Estado, num. 106, de 4 de mayo de 2006.

Ley de Educación de Andalucía del 17/2007 de 10 de diciembre. En Boletín Oficial de la Junta de Andalucía, num. 252, de 26 de diciembre de 2007.

Llopis, C (1996). Hacia una educación global: los temas transversales. Aula de Innovación Educativa, (51), 1923.

Momot, M. (2006). Auschwitz Birkenau, lugar de memoria y museo. Zakopane: Krokus. 
Olmos Moreno, L.I. (2011). Trabajando las competencias en educación secundaria obligatoria desde la obra narrativa juvenil Las lágrimas de Shiva. Espiral. Cuadernos del profesorado [en línea], 4(7), 57-65. Disponible en: http://www.cepcuevasolula.es. Consulta [2011, 1 de mayo].

Rivero, P. (2004). Cómic en el aula de Historia. Apuntes para su creación. Proyecto Clío [en línea], 30. Disponible en: http:/clio.rediris.es/n30/comic.htm. Consulta [2010, 1 de noviembre].

Rodríguez Diéguez, J.L. (1988). El cómic y su utilización didáctica. Los tebeos en la enseñanza. Barcelona: Gustavo Gili.

Spielgman, A. (2007). Maus, retrato de un superviviente. Barcelona: Reservoir Books.

Sarto, M.P. y Venegas, M.E. (2009). Aspectos clave de la educación inclusiva. Salamanca: Publicaciones del INICO [Colección Investigación].

Vera, A.L. y Aguaded, J.I. (2006). Geohistoria.net. Comunicación y tecnologías en la enseñanza de las Ciencias Sociales. Huelva: Grupo Comunicar Ediciones.

Zabala, A. (2008). 11 ideas clave. Cómo aprender y enseñar competencias. Barcelona: Graó.

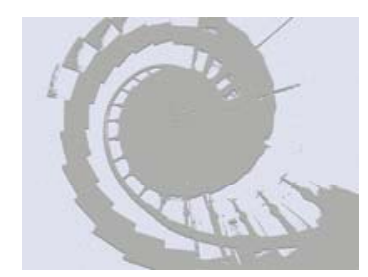

